\title{
Constraining spectral models of a terrestrial gammaray flash from a terrestrial electron beam observation by the AtmosphereSpace Interactions Monitor
}

Sarria, D.; Østgaard, N.; Kochkin, P.; Lehtinen, N.; Mezentsev, A.; Marisaldi, M.; Lindanger, A.; Maiorana, C.; Carlson, B. E.; Neubert, T.

Total number of authors:

22

Published in:

Geophysical Research Letters

Link to article, DOI:

10.1029/2021GL093152

Publication date:

2021

Document Version

Publisher's PDF, also known as Version of record

Link back to DTU Orbit

Citation (APA):

Sarria, D., Østgaard, N., Kochkin, P., Lehtinen, N., Mezentsev, A., Marisaldi, M., Lindanger, A., Maiorana, C., Carlson, B. E., Neubert, T., Reglero, V., Ullaland, K., Yang, S., Genov, G., Qureshi, B. H., BudtzJørgensen, C., Kuvvetli, I., Christiansen, F., Chanrion, O., ... Eyles, C. (2021). Constraining spectral models of a terrestrial gammaray flash from a terrestrial electron beam observation by the AtmosphereSpace Interactions Monitor. Geophysical Research Letters, 48(9), [e2021GL093152]. https://doi.org/10.1029/2021GL093152

\section{General rights}

Copyright and moral rights for the publications made accessible in the public portal are retained by the authors and/or other copyright owners and it is a condition of accessing publications that users recognise and abide by the legal requirements associated with these rights.

- Users may download and print one copy of any publication from the public portal for the purpose of private study or research.

- You may not further distribute the material or use it for any profit-making activity or commercial gain

- You may freely distribute the URL identifying the publication in the public portal 


\section{Geophysical Research Letters}

\author{
RESEARCH LETTER \\ 10.1029/2021GL093152 \\ Key Points: \\ - Observation of a Terrestrial Electron \\ Beam (TEB) with a spectrum \\ resolved down to $50 \mathrm{keV}$ \\ - A method to constrain the energy \\ spectrum of the source Terrestrial \\ Gamma ray Flash (TGF) based on \\ the detection of the associated TEB \\ is presented \\ - Only TGFs originating from fully \\ developed Relativistic Runaway \\ Electron Avalanche models can \\ explain the observation
}

\section{Supporting Information:}

Supporting Information may be found in the online version of this article.

Correspondence to:

D. Sarria,

david.sarria@uib.no

Citation:

Sarria, D., Østgaard, N., Kochkin, P., Lehtinen, N., Mezentsev, A., Marisaldi, M., et al. (2021). Constraining spectral models of a terrestrial gamma-ray flash from a terrestrial electron beam observation by the atmosphere-space interactions monitor. Geophysical Research Letters, 48, e2021GL093152. https://doi.org/10.1029/2021GL093152

Received 2 MAR 2021

Accepted 14 APR 2021
(C) 2021. The Authors.

This is an open access article under the terms of the Creative Commons Attribution-NonCommercial License, which permits use, distribution and reproduction in any medium, provided the original work is properly cited and is not used for commercial purposes.
Constraining Spectral Models of a Terrestrial GammaRay Flash From a Terrestrial Electron Beam Observation by the Atmosphere-Space Interactions Monitor

\author{
D. Sarria ${ }^{1}$ (D), N. Østgaard ${ }^{1}$ (D) P. Kochkin ${ }^{1}$ (D), N. Lehtinen ${ }^{1}$, A. Mezentsev $^{1}$ (D), M. Marisaldi ${ }^{1}$ (D), \\ A. Lindanger ${ }^{1}$ (D) C. Maiorana ${ }^{1}$ (D) B. E. Carlson ${ }^{1,2}$ (D) T. Neubert ${ }^{3}$ (D) V. Reglero ${ }^{4}$, \\ K. Ullaland ${ }^{1}$ (D) S. Yang ${ }^{1}$, G. Genov ${ }^{1}$ (D, B. H. Qureshi ${ }^{1}$, C. Budtz-Jørgensen ${ }^{3}$, I. Kuvvetli ${ }^{3}$ (D), \\ F. Christiansen ${ }^{3}$ (D) O. Chanrion ${ }^{3}$ (D), J. Navarro-González ${ }^{4}$ (D) P. Connel ${ }^{4}$, and C. Eyles ${ }^{4}$ \\ ${ }^{1}$ Birkeland Centre for Space Science, University of Bergen, Bergen, Norway, ${ }^{2}$ Carthage College, Kenosha, WI, USA, \\ ${ }^{3}$ National Space Institute, Technical University of Denmark, Lyngby, Denmark, ${ }^{4}$ University of Valencia, Valencia, Spain
}

\section{Introduction}

Terrestrial Gamma ray Flashes (TGFs) are short bursts of high energy ( $<40 \mathrm{MeV}$ ) photons, produced during thunderstorms. A review of TGFs theory and observations is presented by Dwyer et al. (2012). TGFs were first detected using the BATSE experiment on-board the CGRO spacecraft (Fishman et al., 1994). Later, TGFs were recorded by the satellites RHESSI (Smith et al., 2005), AGILE (Marisaldi et al., 2014), Fermi (Briggs et al., 2010; Roberts et al., 2018), BeppoSAX (Ursi et al., 2017), and the Atmosphere-Space Interactions Monitor (ASIM) (Neubert, Østgaard, Reglero, Blanc, et al., 2019). ASIM was successfully launched and docked to the International Space Station in April 2018 and started science operations since June 2018. The first results from ASIM were presented by Østgaard, Neubert, et al. (2019); Sarria et al. (2019); Neubert, Østgaard, Reglero, Chanrion, et al. (2019).

When referring to "electrons beams" in the context of TGFs, one can think of two different objects. The first is associated with the production process of the TGF. This production process takes place, at least for TGF detectable from space, between $\approx 10$ and $\approx 15 \mathrm{~km}$ altitude. This first type of "electron beam" consists of the 
Relativistic Runaway Electron Avalanche (RREA) producing the TGF's high energy photons. This RREA is not detectable from space since it is impossible for it to go through the atmosphere layer. The second type of "electron beam" is called "terrestrial electron beam" (TEB) and is produced higher in the atmosphere by the TGF's photons, though the processes of Compton scattering and electron-positron pair production. Since electron-positron pair production is involved, TEBs are composed of a fraction of positrons, typically 10\%-30\% (see Briggs et al. (2011), table 1). A TEB is bound ("beamed") around the magnetic field line intercepting the source TGF's geographical location (Cohen et al., 2010; Dwyer et al., 2008; Sarria et al., 2015). Most electrons and positrons forming TEBs are produced above $40 \mathrm{~km}$ altitude, where the air collision frequency of the electrons (and positrons) is comparable to their gyration frequency around geomagnetic field lines. TEBs propagate in space and travel large distances in the magnetosphere. TEBs were first reported from measurements of the CGRO spacecraft (Dwyer et al., 2008). Later, they were detected by Fermi (Briggs et al., 2011; Stanbro et al., 2019), BeppoSAX (Ursi et al., 2017), AGILE (Lindanger et al., 2020), and ASIM (Sarria et al., 2019). RHESSI probably detected one or two TEB event(s), but it has not been $100 \%$ confirmed yet (Gjesteland, 2012; Smith et al., 2006). In general, TEBs are detected much less often than TGFs (e.g., Fermi has a few thousand TGFs and about 30 TEBs) because the detector must be located inside a narrow window of less than a few tens of kilometers along the right geomagnetic field line (intercepting the TGF source position), and they last for only a few milliseconds.

One of the reasons of studying TEBs is to retrieve information about the TGFs that produced them. Briggs et al. (2011) constrained the positron fraction to be between $10 \%$ and $34 \%$, based on three events. Positrons fractions are linked to the spectral shape of the source TGF, as photons with harder spectrums will trigger more electron-positron pair production. In Sarria et al. (2019), the beaming of the source TGF could be constrained between about $30^{\circ}$ and $42^{\circ}$ (half angle, isotropic within a cone). Another reason to study TEBs is that they may have an impact on the inner Van Allen radiation belt, that has not been quantified yet (to our knowledge). Even if it is an important question, it is not the subject of the present paper.

One of the most important question regarding TGFs is their production mechanism. Two main models are proposed to explain the production of TGFs, and in both, the TGF's photons are produced by high energy electrons through the bremsstrahlung process. These high energy electrons form a RREA (Gurevich et al., 1992; Wilson, 1924). In the first model, a large scale electric field within thunderclouds is considered. This requires the presence of initial high energy seed electrons, that may be provided by cosmic-ray secondaries or background radiation. The background electric field is strong enough to produce RREA avalanches, but the RREA mechanism alone is not enough to produce bright enough TGFs (i.e., detectable from space, therefore, with more than $10^{16}$ photons between $50 \mathrm{keV}$ and $40 \mathrm{MeV}$ at source), and a x-ray and positron feedback mechanism is required (the "relativistic feedback"); only possible if large potentials are available (Babich et al., 2005; Dwyer, 2012; Dwyer et al., 2003; Skeltved et al., 2014). This mechanism will produce a discharge of the thundercloud that is of different nature than usual lightning discharges. The resulting high-energy photon spectrum given by this model is a so-called "fully developed" RREA. The development of a RREA process can be characterized by the number of avalanche lengths that were achieved (that depends on the extend and magnitude of the available electric potential). The energy spectrum of the electrons converges to a standard shape $(\approx \exp (-E / 7.3 \mathrm{MeV}))$, which is fully obtained with six or more avalanche lengths, even if the total number of electrons keeps exponentially increasing with the number of avalanche lengths. Another variant of this model uses a lightning leader to push the background (large scale) field above the threshold to trigger the relativistic feedback mechanism (Skeltved et al., 2017).

The second model of TGF production requires a propagating lightning leader. It is sometimes referred as the "leader-streamer" model. It considers that initial seed electrons are produced by the cold runaway mechanism (Gurevich, 1961), happening in the streamer phase or in the leader phase (Celestin \& Pasko, 2011; Chanrion et al., 2014; Dwyer, 2008; Köhn \& Ebert, 2015; Moss et al., 2006). These energetic seed electrons follow a specific distribution and a fraction of them are then accelerated and multiplied by a larger scale electric field, producing a RREA. The larger scale electric field can be the field induced by the leader and/ or a large scale (background) field in the thunderstorm. In principle, leader-based TGF production models do not exclude the possibility of relativistic feedback, that could be more or less important (Skeltved et al., 2017). A parameter that impacts the energy spectrum of emitted photons the most is the potential drop in the leader tip region that is available for the acceleration of energetic electrons. Resulting TGF 


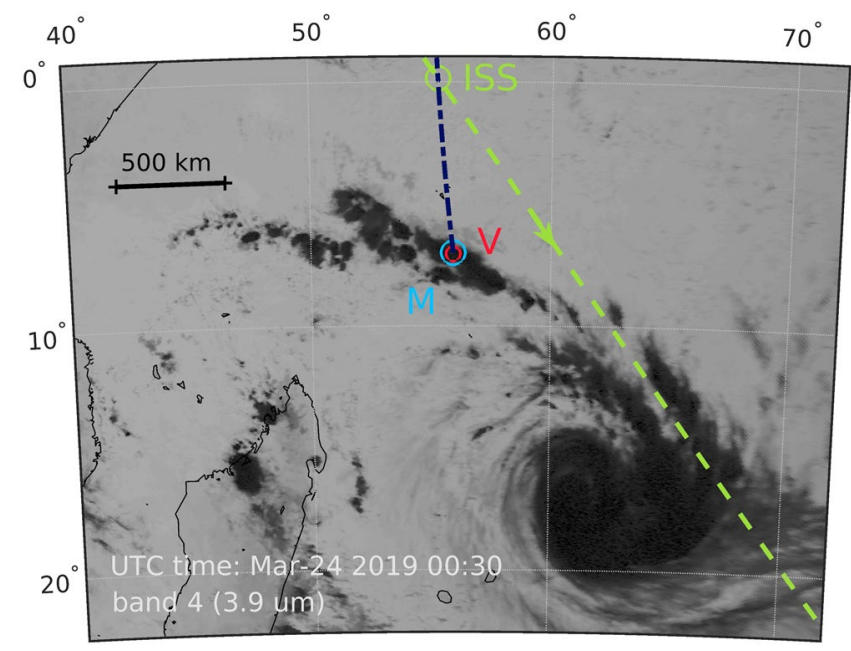

Figure 1. Image from geostationary satellite Meteosat-11 around 00:30 UTC, about $1 \mathrm{~min}$ and $53 \mathrm{~s}$ before the ASIM trigger. The image comes from the optical band 4 ( $3.9 \mu \mathrm{m}, 3 \mathrm{~km}$ resolution). The tropical cyclone Joaninha can be seen in the south-east part of the picture and extends over a thousand of kilometers. The positions of the ISS, the GLD360 match (V), and the magnetic field line footpoint $(\mathrm{M})$ are indicated. The track of the Earth's magnetic field line (dark blue dashed line) and of the trajectory of the ISS (green dashed line) are also showed. Point $V$ is very close to $M$ in both location $(\Delta r=4.82 \mathrm{~km})$ and time $(\Delta t=1.44 \mathrm{~m} \mathrm{~s})$, and is located in the north-western rainbands of the cyclone. ISS, International Space Station. energy spectra for several leader potential drops are presented in Celestin et al. (2015), figure 3. They actually correspond to a more or less developed RREA process. Celestin et al. (2012) also showed that energy spectra harder than the characteristic fully developed RREA spectrum could be achieved by involving non-equilibrium acceleration of electrons. One significant advantage of leader-based TGF models is that they propose an unified approach to explain TGF's X/gamma ray production, as well as x-ray (i.e., softer) emissions from lightning propagating leaders that were observed from ground, balloons, and aircraft (Dwyer et al., 2003, 2004, 2 005, 2011). Mailyan et al. (2019) presented the first study that confronted leader models to TGFs recorded by the Fermi space telescope, with tested potential drops $\leq 200 \mathrm{MV}$. They found that lightning leader models with potentials of $200 \mathrm{MV}$ and tilted beams gave the best fit to the data in most of the analyzed TGF events. However, the range of compatible models is found to be quite wide.

In this article, we report the second TEB event detected by ASIM on March 24, 2019. Compared to the previous event (presented in Sarria et al. (2019)), data from the two detectors are available: the pixelated Low-Energy detector (50-400 keV) and the High Energy Detector (300 keV-30 MeV), that permits an unprecedented spectral analysis of a TEB event. In Section 2, we present the instruments that were used. In Section 3, we present the event. In Section 4, we present the methods and models we use for the spectral analysis. In Section 5, we show the results of the analysis. We conclude in section 6.

\section{Instruments}

The ASIM payload (Neubert, Østgaard, Reglero, Blanc, et al., 2019) consists of two main instruments, the Modular X- and Gamma ray Sensor (MXGS) (Østgaard, Balling, et al., 2019) and the Modular Multi-spectral Imaging Array (MMIA) (Chanrion et al., 2019). ASIM is mounted on the International Space Station (ISS) orbiting the Earth at about $400 \mathrm{kms}$ altitude with an inclination of $51.6^{\circ}$. MXGS consists of two detectors for detecting X- and gamma-rays. The MXGS Low-Energy Detector (LED) is layer of 16,384 pixels of Cadmium-Zink-Telluride (CZT) detector crystals, sensitive to photons with energies from $50 \mathrm{keV}$ to about $400 \mathrm{keV}$. The MXGS High Energy Detector (HED) comprises 12 Bismuth-Germanium-Oxide (BGO) detector modules coupled to photomultiplier tubes (PMT), sensitive in the energy range of $300 \mathrm{keV}$ to about $40 \mathrm{MeV}$.

GLD360 (VAISALA) is a network of ground-based lightning sensors (1-350 kHz) detecting both Cloud-toGround and Intra-Cloud lightning. The GLD360 sensors use a combination of magnetic direction finding and time-of-arrival calculations (from 4 stations or more) to geolocate the lightning source (see acknowledgments for more details). The typical uncertainty on location is about $2.5 \mathrm{~km}$ but it can vary a lot with geographical location (Rudlosky et al., 2017).

We also present data provided by the Meteosat-11 geostationary satellite, that provides regular scans of cloud coverage at several wavelengths (used data comes from band 4, at $3.9 \mu \mathrm{m}$, with a $3 \mathrm{~km}$ spatial resolution). See acknowledgments for more information.

\section{Observation}

Figure 1 shows a map of the event together with Satellite imagery that was provided by the geostationary satellite Meteosat-11. The ASIM trigger UTC time is March 24,2019, 00:31:53.135444 and the ISS was located at latitude of $\phi=0.157^{\circ}$, longitude of $\lambda=55.301^{\circ}$, and altitude of $h=408.6 \mathrm{~km}$, that is above the Indian ocean, close to Madagascar. The ASIM clock has a -20 to $30 \mathrm{~m} \mathrm{~s}$ absolute timing uncertainty with respect to GPS UTC time. A VAISALA (GLD360) discharge event with a UTC time of March 24,2019, 00:31:53.134000 

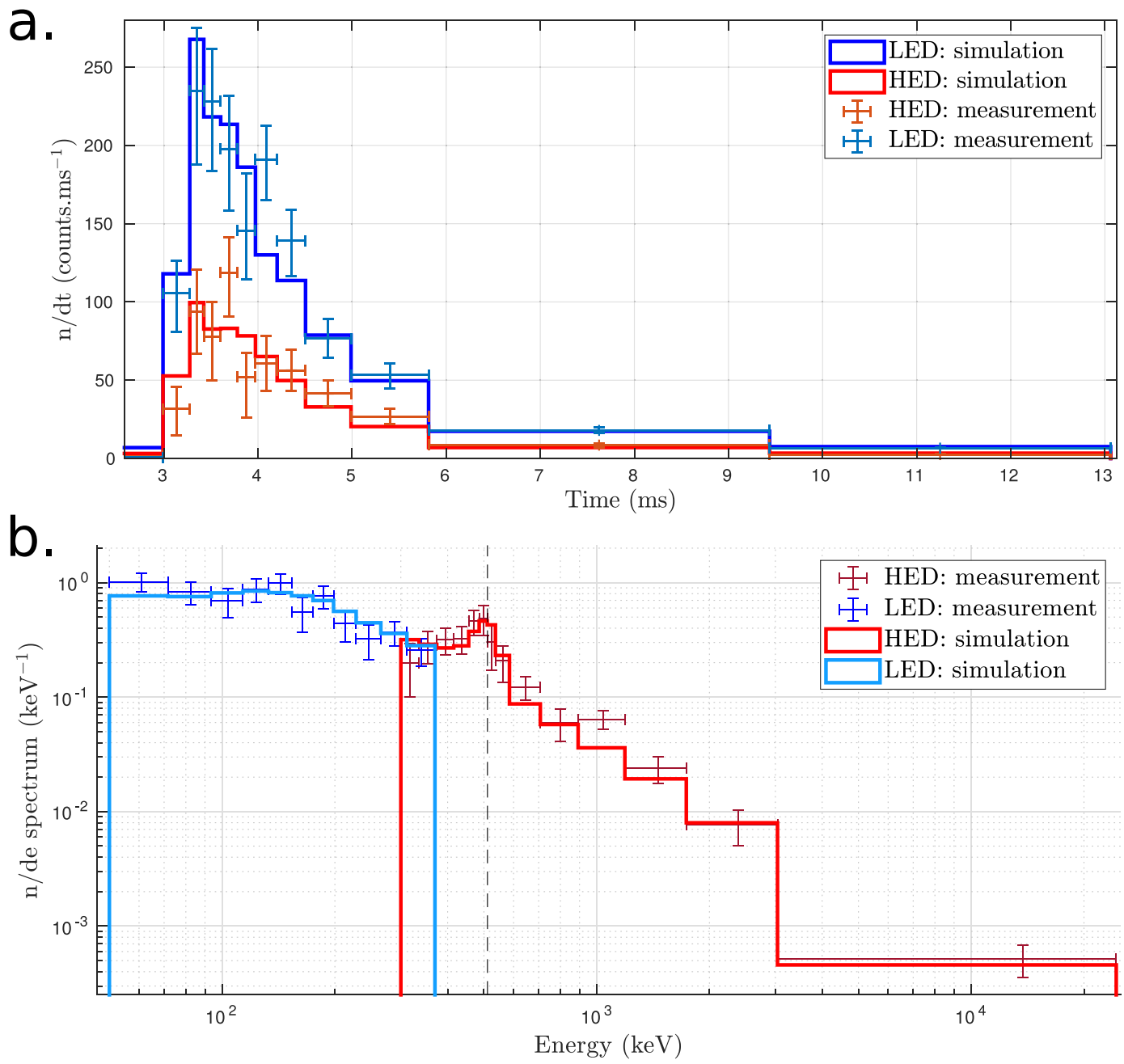

Figure 2. ASIM TEB event 190324. (a) Recorded lightcurve by HED and LED, with $1-\sigma$ Poisson error bars. Simulation results from the consensus model are shown and give a good fit to the data $\left(\chi_{r}^{2}(\mathrm{LED})_{t}=1.33, \chi_{r}^{2}(\mathrm{HED})_{t}=1.34\right)$. (b) Recorded energy spectrum by HED and LED, with $1-\sigma$ Poisson error bars. Simulation results from the consensus model are shown and give a good fit to the data $\left(\chi_{r}^{2}(\mathrm{LED})_{e}=0.88, \chi_{r}^{2}(\mathrm{HED})_{e}=0.85\right)$. ASIM, Atmosphere-Space Interactions Monitor; HED, High Energy Detector; LED, Low Energy Detector; TEB, Terrestrial Electron Beam.

$(\Delta t=1.44 \mathrm{~m} \mathrm{~s}$ ) was found very close to the southern magnetic line footpoint (at $45 \mathrm{~km}$ altitude) intercepting the position of the ISS: $\left[\phi_{G L D 360}=-7.05^{\circ}, \lambda_{G L D 360}=55.91^{\circ}\right]$ and $\left[\phi_{\text {mag, } s}=-7.00^{\circ}, \lambda_{\text {mag,s } s}=55.92^{\circ}\right]$ that gives $\Delta r=4.82 \mathrm{~km}$. Note that the GLD360 location uncertainty can be up to $20 \mathrm{~km}$ for this event, and the uncertainty in the ISS's position is of the same order. The northern magnetic field line footpoint is located at $\left[\phi_{\text {mag }, n}=20.52^{\circ}, \lambda_{m a g, n}=55.10^{\circ}\right]$, but no lightning activity was observed close to it. No lightning activity was detected by GLD360 below the ISS, within $540 \mathrm{~km}$ and $\pm 1 \mathrm{~s}$ around the trigger time. The MMIA photometers did not detect any lightning activity below the ISS as well.

From satellite imagery (Figure 1), it appears that the southern magnetic field line footpoint is located in the rainbands of a tropical cyclone, named "Joaninha." It is the first time that the detection of a TEB associated to a TGF produced in a cyclone is reported.

Figure 2a shows the recorded lightcurves for LED and HED, as well as a modeling result. The latter is obtained using what will be referred as the "consensus model," that assumes a source TGF located at the southern magnetic footpoint, at $12 \mathrm{~km}$ altitude, with an angular distribution following a Gaussian distribution with $\sigma_{\theta}=20^{\circ}$ (centered on zenith), and with an energy spectrum $\propto E^{-1} \exp (-E / 7.3 \mathrm{MeV})$ (maximum energy set to $40 \mathrm{MeV}$ ). More information about the modeling is presented in the next section. The consensus 
model gives a very good fit to the data (see figure label). Figure $2 \mathrm{~b}$ shows the spectra recorded by the MXGS instrument for LED and HED. There are a total of 168 recorded counts in HED and 307 counts in LED. The error bars are $1-\sigma(\approx 68 \%$ interval) assuming Poisson statistics on the count per bin values given by the model. The spectrum shows a line at $511 \mathrm{keV}$, that is expected because the electron beams contains a significant fraction of positrons. The consensus model gives a very good fit to the spectral data as well (see figure label), and indicates a positron to electron ratio of $16.1 \%$. This value is comparable to previous results (Briggs et al., 2011).

\section{Method to Constrain the Source TGF Spectrum}

As presented in the introduction, for any considered TGF production scenario, the spectral shape for the TGF is governed by the RREA process that produces high-energy photons through the bremsstrahlung process. A RREA can be more or less developed depending on how many avalanches lengths have been achieved, that depends on the available potential (in the leader and/or background electric field) and the extend of the electric field(s). The resulting TGF photon energy spectrum can be approximated with Equation 1:

$$
f(E) \propto E^{-1} \exp (-E / \epsilon) \text {, with } E<E_{m}
$$

where $E$ is the energy, $\epsilon$ is a cut-off energy and $E_{m}$ is the maximum allowed energy. TGF energy spectra from fully developed RREA are excepted to have $\epsilon \geq 5 \mathrm{MeV}$ (Dwyer, 2012; Sarria et al., 2018; Skeltved et al., 2014). Typical fully developed TGF spectra used in the literature have $\epsilon=6.5-7.3 \mathrm{MeV}$, and $E_{m}$ of $30-40 \mathrm{MeV}$. TGF production models based on a propagating lightning leader can, in theory, produce bright TGFs (i.e., detectable from space, therefore, with more than $10^{16}$ photons at source) but that shows a partially developed RREA spectrum. This is because, for these models, typically $10^{12}$ (or more) energetic electrons are initially provided by the cold runaway mechanism. Leader models with potential drops as low as $\approx 160$ MV could potentially produce bright TGFs (see Celestin et al. (2015), Table 1). By “potential drop," it is meant the potential difference between the tip of the lightning leader and the ambient potential.

Equation 1 can fit a fully developed RREA (using $\epsilon \geq 5 \mathrm{MeV}, E_{m}=40 \mathrm{MeV}$ ), as well as partially developed RREA energy spectra resulting from leader models. The leader $300 \mathrm{MV}$ model from Celestin et al. (2015) (figure 3) can be fit by Equation 1 with $\epsilon=4.7 \mathrm{MeV}$ and $E_{m}=30 \mathrm{MeV}$. The $160 \mathrm{MV}$ leader model can be fit by Equation 1 using $\epsilon=4.3 \mathrm{MeV}$ and $E_{m}=20 \mathrm{MeV}$. In the cases of potential drops of 160 and $300 \mathrm{MV}$, the initial electron's positions are set at 2 and $3.5 \mathrm{~m}$ from the leader tip, respectively, because of the shielding of the electric field (Skeltved et al., 2017). The corresponding effective electric potential drops (i.e., that the energetic electrons can use) are, respectively, 28 and 53 MV (Celestin et al., 2015).

In addition to the 160 and $300 \mathrm{MV}$ leader spectra, we chose to test spectra with $\epsilon$ equal to $6.5,7.3,8$, and $10 \mathrm{MeV}$ (all using $E_{m}=40 \mathrm{MeV}$ ). The first two values correspond to values used in the literature (Bowers et al., 2017; Dwyer et al., 2012; Sarria et al., 2018; Xu et al., 2019). After looking at the preliminary results using these two values, we decided to add $\epsilon=8 \mathrm{MeV}$ and $\epsilon=10 \mathrm{MeV}$. These last two values were primarily added on an ad hoc basis, but a physical justification is that, in theory, non-uniform electric fields in leader models can also produce TGF spectra harder than typical fully developed RREA if non-uniform electric fields are involved (Celestin et al., 2012). We decided not to test values above $\epsilon=10 \mathrm{MeV}$ and $E_{m}=40 \mathrm{MeV}$, since such high energies seem irrelevant for TGFs, given our currrent undertsanding.

To generate a simulated ASIM spectrum, we proceeded to forward modeling of the recorded spectrum, using a two stage simulation. In the first stage, a TGF is started at $12 \mathrm{~km}$ altitude, assuming one of the initial energy spectra models, and is propagated to the ISS altitude (about $400 \mathrm{~km}$ ) using the Geant4-based Monte-Carlo model presented in Sarria et al. (2019) and publicly available (see acknowledgments). Energy, 3D-momentum, and times of electrons/positrons reaching the ISS within a radius of $80 \mathrm{~km}$ (at ISS altitude) are saved. At the end of this stage, at least 1 million particle records are required for each tested source TGF spectrum model.

In the second stage, the recorded electrons/positrons are used as input of the ASIM mass model to simulate the response of the instrument. A rotation of frame of reference (Earth to ISS) is applied, and we also included the local geomagnetic field. The used mass model includes the ASIM detectors (MXGS, MMIA), 
the instrument platform, as well as non-negligible surrounding elements (e.g., the Columbus module). The energy deposition on the detectors can be direct, that is, electrons/positrons hitting directly a CZT or BGO crystal, or indirect. In the indirect case, electrons/positrons emit bremsstrahlung photons by interaction with the surrounding material that hit at least one crystal. Photons can also come from annihilating positrons, with specific energy of $511 \mathrm{keV}$. For HED, because of the shielding, about $98 \%$ of the energy deposition is due to indirect hits into the BGO crystals. For LED, direct hits are more important: about $72 \%$ of the energy deposition. This explains why the effective area of LED is larger than HED when considering incident electrons/positrons. The effective area is calculated as the geometrical area $\left(\approx 900 \mathrm{~cm}^{2}\right.$ for HED and $\approx 1,024 \mathrm{~cm}^{2}$ for LED) multiplied by the probability of an incident TEB electron to deposit more than $300 \mathrm{keV}$ into at least one BGO crystal (for HED), or more than $50 \mathrm{keV}$ into at least one CZT pixel (for LED).

At the end of the second stage, a simulation data set in the form of a list of detected time and energy counts is generated. To be able to completely neglect the simulation noise, it is required to have at least 1,000,000 counts on each detector to build each energy spectrum and calculate the effective areas. The final modeled spectra also include a background component build from real background data.

A key feature of performing spectral analysis on the TEB, instead of TGF, is that the energy spectrum of the constituting electrons and positrons above $100 \mathrm{~km}$ altitude is only weakly dependent on the following parameters:

- the radial distance between the TEB center and the ISS. The concept of radial is presented more precisely in the supporting information, Figure A.1

- the beaming and the tilt angles of the source TGF

- the source altitude of the TGF, if set between 10 and $15 \mathrm{~km}$

Actually, we found that the spectrum of the source TGF is the dominanting factor that affects the spectrum of the detected TEB. This permit a substantial simplification of the problem as it reduces drastically the number of free parameters to include in the analysis. Since these three points are crucial for this analysis, we provide in the supporting information document more detailed arguments and simulation results supporting those three points. It includes the results of the procedure described below if applied to source TGF altitudes of 10 and $15 \mathrm{~km}$, and various opening angle distributions and tilt angles. The effect of the source TGF altitude is small and does not affect significantly the results presented next (this issue discussed into details in the supporting information, section B). In the following, we fix the source TGF model to a source altitude to $12 \mathrm{~km}$, a Gaussian angular distribution with $\sigma=20^{\circ}$, and no tilt angle.

The simulated spectra are evaluated with respect to the observation, separately for the LED (50-370 keV) and the HED (0.3-40 MeV), and with both detectors together. To compare the modeling results to the observation, we use three methods: a likelihood analysis, a $\chi^{2}$ analysis (Eadie et al., 1971; Lyons, 1986; Martin, 1971), and the effective LED/HED area ratio. Note that these three criteria are not independent as they use the same data sets: the list of measured and simulated energy counts by the HED and the LED.

For the likelihood analysis, a value of $-2 \ln (\mathcal{L})$, the Negative Log-Likelihood, is calculated. The model with the lowest value of $-2 \ln (\mathcal{L})$ is considered to be the best description of the observation. Models are considered to be also possible if their $-2 \ln (\mathcal{L})$ values have a difference of less than a threshold value $\tau$. We calculated that $\tau \approx 5$ for a confidence level of about $99 \%$, similar to the one used by Mailyan et al. (2016) for Fermi-GBM observations. This value assumes that $-2 \ln (\mathcal{L})$ evolves following approximately a normal distribution with respect to the free parameter(s). In the following, we present the values $\Delta_{\text {mle }}$, that are the values of $-2 \ln (\mathcal{L})$ subtracted by the value of $-2 \ln (\mathcal{L})$ for the best model. Therefore, the best model has $\Delta_{m l e}=0$ and compatible models have $\Delta_{m l e} \leq \tau$. A verification if a given model was found worse than another just because of random fluctuations ("by chance") is also performed.

We also provide a reduced $\chi^{2}$ value, noted $\chi_{r}^{2}$. If $\chi_{r}^{2}$ is below a critical value, the model is considered compatible with the measurement, and above the model is considered incompatible. The Pearson's $\chi^{2}$ method is affected by choice of binning (i.e., energy intervals chosen to build the spectra). To mitigate this effect, we chose a binning with at least 7 measurement counts on each bin for HED, and at least 10 for LED. These two binnings are used to make the spectra presented in Figure 2b. Given the used binning, the critical value 
Table 1

Table Summarizing the Comparison of the Tested Spectral Models With the Measurement

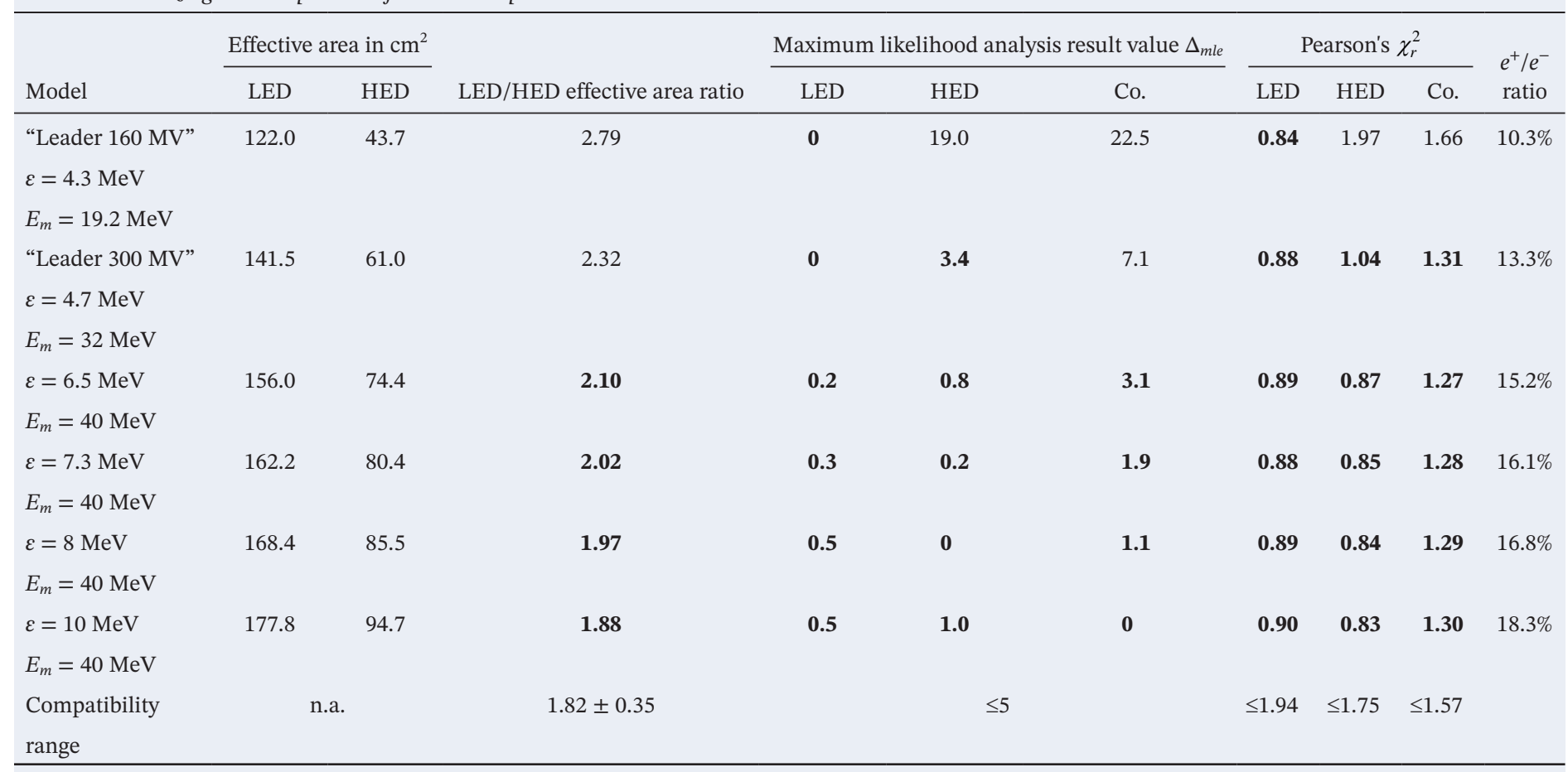

Three main criteria are presented: the led/hed effective area ratio, the maximum likelihood and the Pearson's $\chi_{r}^{2}$. “Co." stands for the led and hed combination. the compatibility range for the different criteria are also indicated. bold values indicate compatible models for the given criteria (column).

Abbreviations: HED, High Energy Detector; LED, Low Energy Detector.

$\chi_{r, c}^{2}$ is 1.94 (8 degrees of freedom) for the LED, 1.75 for the HED ( 12 degrees of freedom), and 1.57 for the combination of both (20 degrees of freedom).

Compared to the Pearson's $\chi^{2}$, the maximum likelihood analysis presents the advantage of not relying on a bining of the measurement data: it keeps all its granularity, that is, no information is lost by binning the measurements. The maximum likelihood analysis is better suited than the $\chi^{2}$ to estimate which model is the best description of the observation (see, e.g., Hauschild \& Jentschel, 2001)

\section{Results and Discussion}

Table 1 summarizes the results of this study. The models are sorted according the prevalence of high energies (also called "hardness") or, equivalently, by decreasing LED/HED effective area ratio. As indicated in the previous section, three main evaluation criteria are presented: the reduced Pearson's $\chi_{r}^{2}$, the maximum likelihood, and the LED/HED effective area ratio.

Concerning the LED spectral fits (Table 1), all the models give good fits, using the $\chi_{r}^{2}$ or the Maximum likelihood analysis. We interpret this as the energy range of 50-370 keV being too narrow to discriminate between the models.

Concerning the HED spectral fits, looking at the $\chi_{r}^{2}$ values, only the $160 \mathrm{MV}$ leader model is found incompatible. This criterion gives similar conclusions when LED and HED spectra are combined.

The maximum likelihood analysis on the HED spectrum shows that the best model is for $\epsilon=8 \mathrm{MeV}$. The fit for $\epsilon=7.3 \mathrm{MeV}$ is also very close. It indicates that the leader $300 \mathrm{MV}$ model and harder spectra are also possible explanations. If LED and HED spectra are combined, the best model is then $\epsilon=10 \mathrm{MeV}$ (but $\epsilon=8 \mathrm{MeV}$ is a very close fit), and only models with $\epsilon=6.5 \mathrm{MeV}$ or greater are compatible. 
Since 307 counts are observed for LED (>50 keV) and 168 for HED (>300 keV), the observed ratio is 1.83 . Considering that the two count numbers individually follow a Poisson statistic (but the ratio does not), the uncertainty on the ratio is \pm 0.35 ( $95 \%$ interval). It implies that, using this criterion, the two leader-based source TGF spectral models (160 and $300 \mathrm{MV}$ ) are incompatible. The effective area ratio analysis indicates that the models with $\epsilon \geq 6.5 \mathrm{MeV}$ are compatible. In particular, we cannot exclude $\epsilon=8$ and $\epsilon=10 \mathrm{MeV}$. This is a similar result as obtained with the maximum likelihood analysis.

For this event, TGF spectra harder than previously expected are not excluded. AGILE did report observations of TGF surprisingly hard (up to $100 \mathrm{MeV}$ ), but they were later found explainable from instrumental effects (Marisaldi et al., 2019). Our analysis does not exclude that the mechanism presented in (Celestin et al., 2012) could not be responsible for producing TGFs with a sigtly harder energy spectra than fully developed RREA.

The results presented in this article are only valid for a single event, and it does not imply that leader models with potentials of $300 \mathrm{MV}$ or less could explain other TGF (and TEB) events. It is also possible that because our method relies on the detection of a TEB, we are biased toward a population of strong TGFs, necessitating fully developed RREAs. TGFs that could originate from non-fully-developed RREAs (leader models) may never (or very rarely) produce a detectable TEB. This question could be addressable in the future, by applying this analysis to more TEB events. We list possibilities of new studies in the next section.

Finally, Table 1 also indicates the positron/electron ratio. The model giving the best fit $(\epsilon=10 \mathrm{MeV})$ gives a ratio of $18.3 \%$, and the range of compatible models give a ratio ranging from $15.2 \%$ to $18.3 \%$. This range is compatible with estimations from the Fermi space telescope team (Briggs et al., 2011).

\section{Conclusions and Future Work}

We reported the observation of a Terrestrial Electron Beam by ASIM on March 24, 2019, originating from the rainbands of the tropical cyclone Johanina. The associated lightning stroke was detected by the GLD360 network (VAISALA) in close temporal association and very close to the ISS's south magnetic field line footpoint. The TEB spectrum was resolved down to $50 \mathrm{keV}$ for the first time, using the low energy detector (LED) of ASIM. A method to constrain the TGF source energy spectrum based on the TEB detection was presented. It relies on a reduction of the number of free parameters (altitude, angular distribution, and radial distance) possible due to TEB's properties. Comprehensive Monte Carlo simulations were performed to reproduce the observation, assuming several energy spectrum shapes of the source TGF. Using three criteria to evaluate the simulation results with respect to the observation (Maximum likelihood, Pearson's $\chi_{r}^{2}$ and LED/HED count ratio), we showed that source TGF with, at least, a fully developed RREA spectrum $\propto E^{-1} \exp (-E / \epsilon)$ (with $\epsilon \geq 6.5 \mathrm{MeV}, E_{m}=40 \mathrm{MeV}$ ) is compatible with the observation. We could not exclude harder models with $\epsilon=8 \mathrm{MeV}\left(E_{m}=40 \mathrm{MeV}\right)$ and $10 \mathrm{MeV}\left(E_{m}=40 \mathrm{MeV}\right)$, that could potentially be explained by non-equilibrium acceleration of energetic electrons in lightning (Celestin et al., 2012).

In the future, we expect that a larger number of events will be processed using the method presented in this article. For ASIM, it will not be possible before several more years of data gathering, since it currently detects about 4 TEB a year, and not all of them present LED data (only turned ON during the night time of the ISS) or enough counts on LED and HED. In principle, the method presented in this article could also be applied/translated to events from the Fermi GBM TGF/TEB catalog (Roberts et al., 2018), that currently contains about 30 TEB events. Fermi GBM has and high energy (BGO-based) detectors that covers an energy range of $\approx 150 \mathrm{keV}$ to $\approx 30 \mathrm{MeV}$. GBM's NaI detectors could also be used in principle (with an energy range of a few keV to $1 \mathrm{MeV}$ ) but no TEB spectrum using it was reported yet. Since TEB events present lower fluxes (counts per second) than TGFs (typically 20 times), it makes the spectral analysis much less challenging than for TGF events: instrumental effects (dead-time, pile-up), affecting TGF analysis, can be mostly (if not totally) ignored for TEB spectral analysis.

\section{Data Availability Statement}

The data presented in this article is available in the following Zenodo repository: https://doi.org/10.5281/zenodo.4264459. 
The Geant4-based model for Terrestrial Gamma ray Flash (TGF) and associated electrons and positrons propagation in Earth atmosphere and environment (magnetic field) is available in the following repository: https://doi.org/10.5281/zenodo.2597039.

\section{Acknowledgments}

This work was supported by the European Research Council under the European Union's Seventh Framework Program (FP7/2007-2013)/ERC grant agreement n. 320839 and the Research Council of Norway under contract 223252/F50 (CoE). ASIM is a mission of ESA's SciSpace Program for scientific utilization of the ISS and non-ISS space exploration platforms and space environment analogs. This study has received funding from the European Union's Horizon 2020 research and innovation program under the Marie Sklodowska-Curie grant agreement SAINT 722337. ASIM was funded through the ESA ELIPS program, through contracts with TERMA and Danish Technical University (DTU) in Denmark, University of Bergen (UB) in Norway and University of Valencia (UV) in Spain. Additional funding was supported by the ESA PRODEX contracts PEA 4000105639 and 4000111397 to DTU and ESA PRODEX contract 4000102100 and by Norwegian Research Council to UB. The ASIM Science Data Center (ASDC) at DTU is supported by PRODEX contract PEA 4000115884 and by PRODEX contract PEA4000123438 at UB. The ASIM Science Data Center and data analysis activities at the UV are supported by the MINECO Research Grants ESP2015- 69909-C5-1-R and ESP2017-86263-C4-1-R.

The authors thank Vaisala Inc. for access to the GLD360 data. For more information, see https://www.vaisala. com/en/products/systems/lightning/ gld 360 .

The authors acknowledge EUMETSAT for making accessible images from the Meteosat-11 geostationary satellite and the MCFETCH service (https://mcfetch. ssec.wisc.edu/) from the Satellite Data Services (SDS) group, at the University of Wisconsin-Madison Space Science and Engineering Center (SSEC), that is responsible for the access, maintenance, and distribution of real-time and archive weather satellite data. The simulations were performed on resources provided by UNINETT Sigma2 - the National Infrastructure for High Performance Computing and Data Storage in Norway, under project no. NN9526K.

\section{References}

Babich, L. P., Donskoy, E. N., Kutsyk, I. M., \& Roussel-Dupré, R. A. (2005). The feedback mechanism of runaway air breakdown. Geophysical Research Letters, 32(9). https://doi.org/10.1029/2004GL021744

Bowers, G. S., Smith, D. M., Martinez-McKinney, G. F., Kamogawa, M., Cummer, S. A., Dwyer, J. R., et al. (2017). Gamma ray signatures of neutrons from a terrestrial gamma ray flash. Geophysical Research Letters, 44(19). https://doi.org/10.1002/2017GL075071

Briggs, M. S., Connaughton, V., Wilson-Hodge, C., Preece, R. D., Fishman, G. J., Kippen, R. M., et al. (2011). Electron-positron beams from terrestrial lightning observed with Fermi GBM. Geophysical Research Letters, 38, L02808. https://doi.org/10.1029/2010GL046259

Briggs, M. S., Fishman, G. J., Connaughton, V., Bhat, P. N., Paciesas, W. S., Preece, R. D., et al. (2010). First results on terrestrial gamma ray flashes from the fermi gamma-ray burst monitor. Journal of Geophysical Research, 115, A07323. https://doi.org/10.1029/2009JA015242

Celestin, S., \& Pasko, V. P. (2011). Energy and fluxes of thermal runaway electrons produced by exponential growth of streamers during the stepping of lightning leaders and in transient luminous events. Journal of Geophysical Research, 116, A03315. https://doi. org/10.1029/2010JA016260

Celestin, S., Xu, W., \& Pasko, V. P. (2012). Terrestrial gamma ray flashes with energies up to $100 \mathrm{MeV}$ produced by nonequilibrium acceleration of electrons in lightning. Journal of Geophysical Research, 117(A5). https://doi.org/10.1029/2012JA017535

Celestin, S., Xu, W., \& Pasko, V. P. (2015). Variability influence and spectrum of high-energy photon bursts produced by lightning leaders. Journal of Geophysical Research: Space Physics, 120(12), 10,712-10,10. https://doi.org/10.1002/2015JA021410

Chanrion, O., Bonaventura, Z., Çinar, D., Bourdon, A., \& Neubert, T. (2014). Runaway electrons from a 'beam-bulk' model of streamer: Application to TGFs. Environmental Research Letters, 9(5), 055003. https://doi.org/10.1088/1748-9326/9/5/055003

Chanrion, O., Neubert, T., Lundgaard Rasmussen, I., Stoltze, C., Tcherniak, D., Jessen, N. C., et al. (2019). The Modular Multispectral Imaging Array (MMIA) of the ASIM payload on the international space station. Space Science Reviews, 215, 28. https://doi.org/10.1007/ s11214-019-0593-y

Cohen, M. B., Inan, U. S., Said, R. K., Briggs, M. S., Fishman, G. J., Connaughton, V., \& Cummer, S. A. (2010). A lightning discharge producing a beam of relativistic electrons into space. Geophysical Research Letters, 37(18), L18806. https://doi.org/10.1029/2010GL044481

Dwyer, J. R. (2008). Source mechanisms of terrestrial gamma-ray flashes. Journal of Geophysical Research, 113(D10), D10103. https://doi. org/10.1029/2007JD009248

Dwyer, J. R. (2012). The relativistic feedback discharge model of terrestrial gamma ray flashes. Journal of Geophysical Research, 117(A2), A02308. https://doi.org/10.1029/2011JA017160

Dwyer, J. R., Grefenstette, B. W., \& Smith, D. M. (2008). High-energy electron beams launched into space by thunderstorms. Geophysical Research Letters, 35, L02815. https://doi.org/10.1029/2007GL032430

Dwyer, J. R., Rassoul, H. K., Al-Dayeh, M., Caraway, L., Chrest, A., Wright, B., et al. (2005). X-ray bursts associated with leader steps in cloud-to-ground lightning. Geophysical Research Letters, 32(1). https://doi.org/10.1029/2004gl021782

Dwyer, J. R., Rassoul, H. K., Al-Dayeh, M., Caraway, L., Wright, B., Chrest, A., et al. (2004). Measurements of X-ray emission from rocket-triggered lightning. Geophysical Research Letters, 31(5). https://doi.org/10.1029/2003gl018770

Dwyer, J. R., Schaal, M., Rassoul, H. K., Uman, M. A., Jordan, D. M., \& Hill, D. (2011). High-speed X-ray images of triggered lightning dart leaders. Journal of Geophysical Research, 116(D15), D20208. https://doi.org/10.1029/2011JD015973

Dwyer, J. R., Smith, D. M., \& Cummer, S. A. (2012). High-energy atmospheric physics: Terrestrial gamma-ray flashes and related phenomena. Space Science Reviews, 173(1-4), 133-196. https://doi.org/10.1007/s11214-012-9894-0

Dwyer, J. R., Uman, M. A., Rassoul, H. K., Al-Dayeh, M., Caraway, L., Jerauld, J., et al. (2003). Energetic radiation produced during rocket-triggered lightning. Science, 299, 694-697. https://doi.org/10.1126/science.1078940

Eadie, W. T., Drijard, D., James, F. E., Roos, M., \& Sadoulet, B. (1971). Statistical methods in experimental physics. Amsterdam, North-Holland. Retrieved from https://cds.cern.ch/record/100342

Fishman, G. J., Bhat, P. N., Mallozzi, R., Horack, J. M., Koshut, T., Kouveliotou, C., et al. (1994). Discovery of intense gamma-ray flashes of atmospheric origin. Science, 264(5163), 1313-1316. https://doi.org/10.1126/science.264.5163.1313

Gjesteland, T. (2012). Properties of terrestrial gamma ray flashes. Modeling and analysis of BATSE and RHESSI data (PhD thesis), The University of Bergen. Retrieved from http://bora.uib.no/handle/1956/6203

Gurevich, A. V. (1961). On the theory of runaway electrons. Soviet Physics Journal of Experimental and Theoretical Physics, 12(5), 904-912.

Gurevich, A. V., Milikh, G. M., \& Roussel-Dupre, R. (1992). Runaway electron mechanism of air breakdown and preconditioning during a thunderstorm. Physics Letters A, 165(5-6), 463-468. https://doi.org/10.1016/0375-9601(92)90348-p

Hauschild, T., \& Jentschel, M. (2001). Comparison of maximum likelihood estimation and chi-square statistics applied to counting experiments. Nuclear Instruments and Methods in Physics Research Section A: Accelerators, Spectrometers, Detectors and Associated Equipment, 457(1), 384-401. https://doi.org/10.1016/s0168-9002(00)00756-7. Retrieved from http://www.sciencedirect.com/science/article/ pii/S0168900200007567

Köhn, C., \& Ebert, U. (2015). Calculation of beams of positrons, neutrons, and protons associated with terrestrial gamma ray flashes. Journal of Geophysical Research - D: Atmospheres, 120(4), 1620-1635. https://doi.org/10.1002/2014JD022229

Lindanger, A., Marisaldi, M., Maiorana, C., Sarria, D., Albrechtsen, K., Østgaard, N., et al. (2020). The 3rd AGILE terrestrial gamma ray flash catalog. Part I: Association to lightning sferics. Journal of Geophysical Research - D: Atmospheres, 125(11), e2019JD031985. https:// doi.org/10.1029/2019JD031985

Lyons, L. (1986). Statistics for nuclear and particle physicists. https://doi.org/10.1017/cbo9781139167710

Mailyan, B. G., Briggs, M. S., Cramer, E. S., Fitzpatrick, G., Roberts, O. J., Stanbro, M., et al. (2016). The spectroscopy of individual terrestrial gamma-ray flashes: Constraining the source properties. Journal of Geophysical Research: Space Physics, 121(A10), 346, 11. https:// doi.org/10.1002/2016JA022702

Mailyan, B. G., Xu, W., Celestin, S., Briggs, M. S., Dwyer, J. R., Cramer, E. S., et al. (2019). Analysis of individual terrestrial gamma-ray flashes with lightning leader models and fermi gamma-ray burst monitor data. Journal of Geophysical Research: Space Physics, 124(8), 71707183. https://doi.org/10.1029/2019ja026912. Retrieved from https://agupubs.onlinelibrary.wiley.com/doi/abs/10.1029/2019JA026912 
Marisaldi, M., Fuschino, F., Tavani, M., Dietrich, S., Price, C., Galli, M., et al. (2014). Properties of terrestrial gamma ray flashes detected by AGILE MCAL below 30 MeV. Journal of Geophysical Research: Space Physics, 119, 1337-1355. https://doi.org/10.1002/2013JA019301

Marisaldi, M., Galli, M., Labanti, C., Østgaard, N., Sarria, D., Cummer, S. A., et al. (2019). On the High-Energy Spectral Component and Fine Time Structure of Terrestrial Gamma Ray Flashes. Journal of Geophysical Research - D: Atmospheres, 124(14), 7484-7497. https:// doi.org/10.1029/2019jd030554. Retrieved 2021-01-22, from https://onlinelibrary.wiley.com/doi/abs/10.1029/2019JD030554

Martin, B. R. (1971). Statistics for physicists [by] B. R. Martin. Academic Press London, New York.

Moss, G. D., Pasko, V. P., Liu, N., \& Veronis, G. (2006). Monte Carlo model for analysis of thermal runaway electrons in streamer tips in transient luminous events and streamer zones of lightning leaders. Journal of Geophysical Research, 111, 2307. https://doi. org/10.1029/2005JA011350

Neubert, T., Østgaard, N., Reglero, V., Blanc, E., Chanrion, O., Oxborrow, C. A., et al. (2019). The ASIM mission on the international space station. Space Science Reviews, 215(2), 26. https://doi.org/10.1007/s11214-019-0592-z. Retrieved 2021-01-22, from http://link.springer. com/10.1007/s11214-019-0592-z

Neubert, T., Østgaard, N., Reglero, V., Chanrion, O., Heumesser, M., Dimitriadou, K., et al. (2019). A terrestrial gamma-ray flash and ionospheric ultraviolet emissions powered by lightning. Science, 367, 183, 186, eaax3872. Retrieved 2021-01-20, from https://doi. org/10.1126/science.aax3872

Østgaard, N., Balling, J. E., Bjørnsen, T., Brauer, P., Budtz-Jørgensen, C., Bujwan, W., et al. (2019). The Modular X- and Gamma-Ray Sensor (MXGS) of the ASIM payload on the international space station. Space Science Reviews, 215, 23. https://doi.org/10.1007/ s11214-018-0573-7

Østgaard, N., Neubert, T., Reglero, V., Ullaland, K., Yang, S., Genov, G., et al. (2019). First 10 Months of TGF Observations by ASIM. Journal of Geophysical Research - D: Atmospheres, 124(24), 14024-14036. https://doi.org/10.1029/2019jd031214. Retrieved 2021-01-20, from https://onlinelibrary.wiley.com/doi/10.1029/2019JD031214

Roberts, O. J., Fitzpatrick, G., Stanbro, M., McBreen, S., Briggs, M. S., Holzworth, R. H., et al. (2018). The first fermi -GBM terrestrial gamma ray flash catalog. Journal of Geophysical Research: Space Physics, 123, 4381-4401. https://doi.org/10.1029/2017JA024837

Rudlosky, S. D., Peterson, M. J., \& Kahn, D. T. (2017). GLD360 Performance Relative to TRMM LIS. Journal of Atmospheric and Oceanic Technology, 34(6), 1307-1322. https://doi.org/10.1175/JTECH-D-16-0243.1

Sarria, D., Blelly, P.-L., \& Forme, F. (2015). MC-PEPTITA: A Monte Carlo model for photon, electron and positron tracking in terrestrial atmosphere-application for a terrestrial gamma ray flash. Journal of Geophysical Research: Space Physics, 120(5), 3970-3986. https://doi. org/10.1002/2014ja020695. Retrieved from https://agupubs.onlinelibrary.wiley.com/doi/abs/10.1002/2014JA020695

Sarria, D., Kochkin, P., Østgaard, N., Lehtinen, N., Mezentsev, A., Marisaldi, M., et al. (2019). The first terrestrial electron beam observed by the atmosphere-space interactions monitor. Journal of Geophysical Research: Space Physics, 124(12), 10497-10511. https://doi. org/10.1029/2019JA027071

Sarria, D., Rutjes, C., Diniz, G., Luque, A., Ihaddadene, K. M. A., Dwyer, J. R., et al. (2018). Evaluation of Monte Carlo tools for high-energy atmospheric physics II: Relativistic runaway electron avalanches. Geoscientific Model Development, 11, 4515-4535. https://doi. org/10.5194/gmd-11-4515-2018

Skeltved, A. B., Østgaard, N., Carlson, B., Gjesteland, T., \& Celestin, S. (2014). Modeling the relativistic runaway electron avalanche and the feedback mechanism with GEANT4. Journal of Geophysical Research: Space Physics, 119, 9174-9191. https://doi. org/10.1002/2014JA020504

Skeltved, A. B., Østgaard, N., Mezentsev, A., Lehtinen, N., \& Carlson, B. (2017). Constraints to do realistic modeling of the electric field ahead of the tip of a lightning leader. Journal of Geophysical Research - D: Atmospheres, 122(15), 8120-8134. https://doi.org/10.1002/ 2016jd026206. Retrieved from https://agupubs.onlinelibrary.wiley.com/doi/abs/10.1002/2016JD026206

Smith, D. M., Grefenstette, B. W., Splitt, M., Lazarus, S. M., Rassoul, H. K., Coleman, L. M., et al. (2006). The anomalous terrestrial gamma-ray flash of 17 January 2004. AGU Fall Meeting Abstracts, AE31A-1040.

Smith, D. M., Lopez, L. I., Lin, R. P., \& Barrington-Leigh, C. P. (2005). Terrestrial gamma-ray flashes observed up to 20 MeV. Science, 307 , 1085-1088. https://doi.org/10.1126/science.1107466

Stanbro, M., Briggs, M. S., Roberts, O. J., Cramer, E., Dwyer, J. R., Holzworth, R. H., et al. (2019). A fermi gamma-ray burst monitor event observed as a terrestrial gamma-ray flash and terrestrial electron beam. Journal of Geophysical Research: Space Physics, 124(12), 1058010591. https://doi.org/10.1029/2019ja026749. Retrieved from https://agupubs.onlinelibrary.wiley.com/doi/abs/10.1029/2019JA026749

Ursi, A., Guidorzi, C., Marisaldi, M., Sarria, D., \& Frontera, F. (2017). Terrestrial gamma-ray flashes in the BeppoSAX data archive. Journal of Atmospheric and Solar-Terrestrial Physics, 156, 50-56. https://doi.org/10.1016/j.jastp.2017.02.014

Wilson, C. T. R. (1924). The electric field of a thundercloud and some of its effects. Proceedings of the Physical Society, London, 37, 32D-37D. https://doi.org/10.1088/1478-7814/37/1/314

Xu, W., Celestin, S., Pasko, V. P., \& Marshall, R. A. (2019). Compton Scattering Effects on the Spectral and Temporal Properties of Terrestrial Gamma-Ray Flashes. Journal of Geophysical Research: Space Physics, 124(8), 7220-7230. https://doi.org/10.1029/2019ja026941. Retrieved from https://agupubs.onlinelibrary.wiley.com/doi/abs/10.1029/2019JA026941 